\title{
THE LEAST EIGENVALUE OF THE SIGNLESS LAPLACIAN OF NON-BIPARTITE UNICYCLIC GRAPHS WITH $K$ PENDANT VERTICES*
}

\author{
RUIFANG LIU $^{\dagger}$, HAIXIA WAN ${ }^{\ddagger}$, JINJIANG YUAN $\ddagger$, AND HUICAI JIA ${ }^{\S}$
}

\begin{abstract}
Let $\mathcal{U}(n, k)$ be the set of non-bipartite unicyclic graphs with $n$ vertices and $k$ pendant vertices, where $n \geq 4$. In this paper, the unique graph with the minimal least eigenvalue of the signless Laplacian among all graphs in $\mathcal{U}(n, k)$ is determined. Furthermore, it is proved that the minimal least eigenvalue of the signless Laplacian is an increasing function in $k$. Let $\mathcal{U}_{n}$ denote the set of non-bipartite unicyclic graphs on $n$ vertices. As an application of the above results, the unique graph with the minimal least eigenvalue of the signless Laplacian among all graphs in $\mathcal{U}_{n}$ is characterized, which has recently been proved by Cardoso, Cvetković, Rowlinson, and Simić.
\end{abstract}

Key words. Non-bipartite unicyclic graph, Signless Laplacian, Least eigenvalue, Pendant vertices.

AMS subject classifications. 05C50.

1. Introduction. All graphs considered are simple, undirected, and connected. The vertex set and edge set of the graph $G$ are denoted by $V(G)$ and $E(G)$, respectively. The distance between vertices $u$ and $v$ of a graph $G$ is denoted by $d_{G}(u, v)$. The degree of a vertex $v$, written by $d_{G}(v)$ or $d(v)$, is the number of edges incident with $v$. A pendant vertex is a vertex of degree 1 . The set of the neighbors of a vertex $v$ is denoted by $N_{G}(v)$ or $N(v)$. The girth $g(G)$ of a graph $G$ is the length of the shortest cycle in $G$, with the girth of an acyclic graph being infinite. Denote by $C_{n}$ and $P_{n}$ the cycle and the path, respectively, on $n$ vertices.

The adjacency matrix of $G$ is defined to be the matrix $A(G)=\left(a_{i j}\right)$, where $a_{i j}=1$ if $v_{i}$ is adjacent to $v_{j}$, and $a_{i j}=0$ otherwise. The degree matrix of $G$ is denoted by $D(G)=\operatorname{diag}\left(d_{G}\left(v_{1}\right), d_{G}\left(v_{2}\right), \ldots, d_{G}\left(v_{n}\right)\right)$. The matrix $Q(G)=D(G)+A(G)$ is called the signless Laplacian or the $Q$-matrix of $G$. Note that $Q(G)$ is nonnegative, symmetric and positive semidefinite, so its eigenvalues are real and can be arranged

${ }^{*}$ Received by the editors on July 19, 2012. Accepted for publication on April 3, 2013. Handling Editor: Bryan L. Shader.

†School of Mathematics and Statistics, Zhengzhou University, Zhengzhou, Henan 450001, China (rfliu@zzu.edu.cn). Supported by the National Natural Science Foundation of China (No. 11201432) and the China Postdoctoral Science Foundation (No. 2011M501185 and 2012T50636).

$\ddagger$ School of Mathematics and Statistics, Zhengzhou University, Zhengzhou, Henan 450001, China.

$\S$ College of Science, Henan Institute of Engineering, Zhengzhou, Henan 451191, China. Supported by the Natural Science Foundation of Education Ministry of Henan Province (No. 13B110939). 
in non-increasing order as follows:

$$
q_{1}(G) \geq q_{2}(G) \geq \cdots \geq q_{n}(G) \geq 0,
$$

where $q_{1}(G)$ is the signless Laplacian spectral radius of graph $G$, and the least eigenvalue $q_{n}(G)$, denoted by $q(G)$ or $q$ for short, is called the least eigenvalue of the signless Laplacian or the least $Q$-eigenvalue of $G$. It is well known 3 , that $q(G)=0$ of a connected graph $G$ if and only if $G$ is bipartite.

Recently, the signless Laplacian matrix of $G$ has received much attention. As pointed out by Haemers and Spence [7, sometimes the matrix $Q$ is more informative about $G$ than the adjacency matrix $A$ or the Laplacian matrix $L(G)=D-A$. Computer investigations of graphs with up to 11 vertices 4 suggest that the spectrum of $D+A$ performs better than the spectrum of $A$ or $D-A$ in distinguishing nonisomorphic graphs.

There has been a lot of work on the signless Laplacian spectral radius of a graph in recent years, however relatively few results on the least eigenvalue of the signless Laplacian $q(G)$ have appeared in the literature. In [8], Li and Wang proposed the following problem concerning the least eigenvalue of the signless Laplacian:

Given a set of graphs $\mathcal{G}$, find a lower bound for the least eigenvalue of the signless Laplacian and characterize the graphs in which the minimal least eigenvalue of the signless Laplacian is attained.

The above problem is actually one of the signless Laplacian version of the classical Brualdi-Solheid problem 11 for the adjacency matrix. Cardoso et al. 2 determined the unique graph with the minimum value of the least eigenvalue of the signless Laplacian of a connected non-bipartite graph with a prescribed number of vertices. Fan et al. 6] minimized the least eigenvalue among all nonsingular unicyclic mixed graphs in the setting of Laplacian of mixed graphs. Their result can be applied to signless Laplacian of graphs directly. Li and Wang [8] characterized the unique graph whose least eigenvalue of the signless Laplacian attains the minimum among all graphs in the complements of trees on $n$ vertices. In [9], Wang and Fan minimized the least eigenvalue of the signless Laplacian among the class of connected graphs with fixed order which contains a given non-bipartite graph as an induced subgraph. In this paper, we focus on the same question for $\mathcal{U}(n, k)$, the set of non-bipartite unicyclic graphs with $n$ vertices and $k$ pendant vertices.

A connected graph is said to be non-bipartite unicyclic, if it has a unique odd cycle, and the same number of vertices and edges. Let $\triangle_{n}^{k}$ be the non-bipartite unicyclic graph obtained from $C_{3}$ and a star $K_{1, k}$ by joining the center of $K_{1, k}$ and a vertex of $C_{3}$ by the path of length $n-k-3$ (see Fig. 1.1). The main result of this 
paper is as follows:

THEOREM 1.1. Let $U^{*}$ have the minimal least eigenvalue of the signless Laplacian among all graphs in $\mathcal{U}(n, k)$. Then $U^{*}$ is isomorphic to $\triangle_{n}^{k}$ for $1 \leq k \leq n-3$. Furthermore, the minimal least eigenvalue of the signless Laplacian $q\left(\triangle_{n}^{k}\right)$ is an increasing function on $k$, i.e., $q\left(\triangle_{n}^{k}\right)<q\left(\triangle_{n}^{k+1}\right)$ for $1 \leq k \leq n-4$.

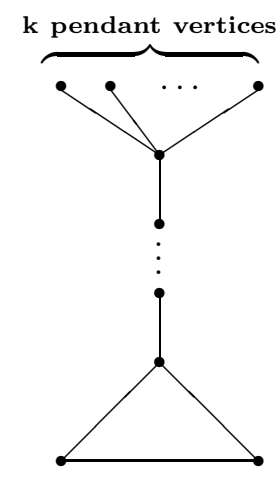

FIG. 1.1. Graph $\triangle_{n}^{k}$.

2. Preliminaries. Denote the least eigenvalue of $Q(G)$ by $q(G)$. The corresponding eigenvectors are called the least $Q$-eigenvectors of graph $G$. Let $X=$ $\left(x_{1}, x_{2}, \ldots, x_{n}\right)^{T} \in \mathbb{R}^{n}$, and $G$ be a graph on vertices $v_{1}, v_{2}, \ldots, v_{n}$. Then $X$ can be considered as a function defined on $G$, that is, each vertex $v_{i}$ is mapped to $x_{i}=x_{v_{i}}$. If $X$ is an eigenvector of $Q(G)$, then it is naturally defined on $V(G)$, where $x_{v}$ is the entry of $X$ corresponding to vertex $v$. One can find that

$$
X^{T} Q(G) X=\sum_{u v \in E(G)}\left(x_{u}+x_{v}\right)^{2} .
$$

Then $q$ is a signless Laplacian eigenvalue of $G$ corresponding to the eigenvector $X$ if and only if $X \neq 0$ and for each vertex $v \in V(G)$,

$$
\left(q-d_{G}(v)\right) x_{v}=\sum_{u \in N_{G}(v)} x_{u} .
$$

In addition, by the Rayleigh-Ritz Theorem, for an arbitrary unit vector $X \in \mathbb{R}^{n}$,

$$
q(G)=\min \left(X^{T} Q(G) X\right) \leq X^{T} Q(G) X
$$

with equality if and only if $X$ is an eigenvector corresponding to the least $Q$-eigenvalue $q(G)$. 
Before giving the proof of Theorem 1.1, we introduce some lemmas in this section.

LEMMA 2.1. ([5]) Let $G$ be a connected non-bipartite graph with minimal degree $\delta$, then $0<q(G)<\delta$. In particular, if $G$ contains a pendant vertex, then $0<q(G)<1$.

Let $G_{1}, G_{2}$ be two vertex-disjoint nontrivial connected graphs with $v_{1} \in V\left(G_{1}\right)$ and $u \in V\left(G_{2}\right)$. The coalescence of $G_{1}$ and $G_{2}$, denoted by $G_{1} v_{1} u G_{2}$, is obtained from $G_{1}$ and $G_{2}$ by identifying $v_{1}$ with $u$ (see Fig. 2.1), where $G_{1}$ and $G_{2}$ are called branches of $G_{1} v_{1} u G_{2}$ with roots $v_{1}$ and $u$, respectively. Let $X$ be a vector defined on $V(G)$. A branch $H$ of $G$ is called a zero branch with respect to $X$ if $x_{v}=0$ for all $v \in V(H)$, otherwise it is called a nonzero branch with respect to $X$.
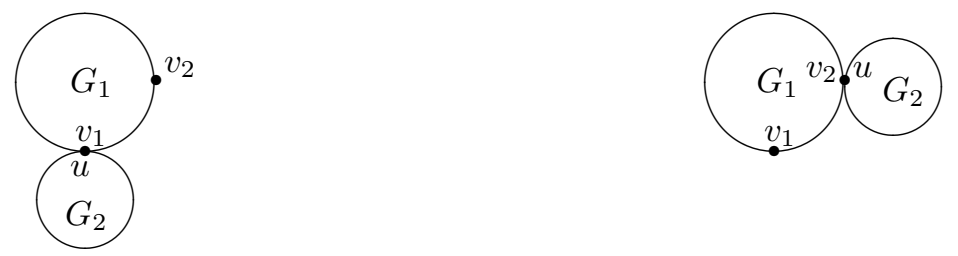

FIG. 2.1. $G_{1} v_{1} u G_{2}$ and $G_{1} v_{2} u G_{2}$.

Lemma 2.2. ([9]) Let $G_{1}, G_{2}$ be two vertex-disjoint nontrivial connected graphs with $v_{1}, v_{2} \in V\left(G_{1}\right)$ and $u \in V\left(G_{2}\right)$. Let $X$ be a least $Q$-eigenvector of $G_{1} v_{1} u G_{2}$. If $\left|x_{v_{1}}\right| \leq\left|x_{v_{2}}\right|$, then

$$
q\left(G_{1} v_{1} u G_{2}\right) \geq q\left(G_{1} v_{2} u G_{2}\right)
$$

with equality only if $\left|x_{v_{1}}\right|=\left|x_{v_{2}}\right|$ and $d_{G_{2}}(u) x_{u}=-\sum_{v \in N_{G_{2}}(u)} x_{v}$.

LEMma 2.3. ([9]) Let $G$ be a nontrivial non-bipartite connected graph, and let $G_{k, l}$ be the graph obtained by coalescing $G$ with two paths $P_{k+1}$ and $P_{l+1}$ by identifying an end vertex of $P_{k+1}$ and an end vertex of $P_{l+1}$ both with the same vertex $v$ of $G$. If $k \geq l \geq 1$, then

$$
q\left(G_{k, l}\right) \geq q\left(G_{k+1, l-1}\right)
$$

with strict inequality if $x_{v} \neq 0$, where $X$ is a least $Q$-eigenvector of $G_{k, l}$.

LEMMA 2.4. ([9]) Let $G$ be a connected graph which contains a bipartite branch $H$ with root $w$. Let $X$ be a least $Q$-eigenvector of $G$.

(i) If $x_{w}=0$, then $H$ is a zero branch of $G$ with respect to $X$.

(ii) If $x_{w} \neq 0$, then $x_{v} \neq 0$ for every vertex $v \in V(H)$. Furthermore, for every vertex $v \in V(H), x_{v} x_{w}$ is either positive or negative, depending on whether $v$ is or is not in the same part of the bipartite graph $H$ as w; consequently, $x_{u} x_{v}<0$ for each edge 
$u v \in E(H)$.

Lemma 2.5. ([9]) Let $G$ be a connected non-bipartite graph, and let $X$ be a least $Q$-eigenvector of $G$. Let $T$ be a tree, which is a nonzero branch of $G$ with respect to $X$ and with root $w$. Then $\left|x_{u}\right|<\left|x_{v}\right|$ whenever $u, v$ are vertices of $T$ such that $u$ lies on the unique path from $w$ to $v$.

Let $\mathcal{U}_{n}$ denote the set of non-bipartite unicyclic graphs on $n$ vertices. For any $U \in \mathcal{U}_{n}$, let $C_{g}$ be the unique odd cycle in $U$, where $g$ is the girth of $U$. In [2, Cardoso et al. obtained the following important result.

LEMMA 2.6. ([2]) Let $U$ be a non-bipartite unicyclic graph on $n$ vertices. Let $X$ be a least $Q$-eigenvector of $U$. Then

(i) $x_{s} x_{t} \geq 0$ for some edge st of $C_{g}$;

(ii) $x_{u} x_{v} \leq 0$ for any other edge uv of $U$;

(iii) if $x_{s} x_{t}=0$, then either $x_{s}$ or $x_{t}$ is nonzero;

(iv) if $u$ is a vertex of $U$ other that $s$ or t, then $\left|x_{u}\right|>\left|x_{s}\right|$ or $\left|x_{u}\right|>\left|x_{t}\right|$.

3. Characterization of the extremal graph. Let $\mathcal{U}(n, k)$ be the set of nonbipartite unicyclic graphs with $n$ vertices and $k$ pendant vertices, where $n \geq 4$. Let $U^{*}$ have the minimal least $Q$-eigenvalue in $\mathcal{U}(n, k)$. By Lemma 2.1. clearly $0<q\left(U^{*}\right)<1$. Let $\triangle_{n}^{k} \in \mathcal{U}(n, k)$ be the non-bipartite unicyclic graph obtained from $C_{3}$ and a star $K_{1, k}$ by joining the center of $K_{1, k}$ and a vertex of $C_{3}$ by the path of length $n-k-3$.

First, we consider the case of $k=n-3$.

Theorem 3.1. Let $U^{*}$ have the minimal least $Q$-eigenvalue in $\mathcal{U}(n, n-3)$. Then $U^{*}$ is isomorphic to $\triangle_{n}^{n-3}$.

Proof. For $U^{*} \in \mathcal{U}(n, n-3)$, then the non-bipartite unicyclic graph $U^{*}$ is obtained from $C_{3}$ by adding some pendant edges to its vertices. Suppose that $U^{*}$ is not isomorphic to $\triangle_{n}^{n-3}$, then there exist two vertices $u, v$ on $C_{3}$ in $U^{*}$, which have $k_{1}, k_{2}$ pendant edges, respectively. Let $w$ be a vertex of $C_{3}$ other than $u$ or $v$. Let $X$ be a unit $Q$-eigenvector of $U^{*}$ corresponding to $q\left(U^{*}\right)$, without loss of generality, we can assume that $\left|x_{u}\right| \geq\left|x_{v}\right|$. Then by Lemma 2.2, $q\left(U^{*}\right) \geq q(U)$, where $U$ is obtained from $U^{*}$ by shifting $k_{2}$ pendant edges from vertex $v$ to vertex $u$. If the equality holds, by Lemma 2.2, $\left|x_{u}\right|=\left|x_{v}\right|$ and $k_{2} x_{v}=-k_{2} x_{s}$, where $s \in N_{U^{*}}(v) \backslash\{u, w\}$. The eigenvalue equations at $v$ and $w$ of $U^{*}$ yield

$$
\left(q-k_{2}-2\right) x_{v}=k_{2} x_{s}+x_{u}+x_{w},
$$

$$
(q-2) x_{w}=x_{u}+x_{v}
$$


where $q=q\left(U^{*}\right)$. Since $k_{2} x_{v}=-k_{2} x_{s}$, (3.1) implies that

$$
(q-2) x_{v}=x_{u}+x_{w} .
$$

Note that $x_{v} \neq 0$ (otherwise $X=0$ by Lemma 2.2 and the eigenvalue equation (2.1)). If $x_{u}=x_{v}$, then we can deduce that $q\left(U^{*}\right)=1$ or $q\left(U^{*}\right)=4$ from (3.2) and (3.3), a contradiction. If $x_{u}=-x_{v}$, then we can obtain that $q\left(U^{*}\right)=1$ from(3.2) and (3.3), a contradiction. Thus, there exists $U \in \mathcal{U}(n, n-3)$ such that $q\left(U^{*}\right)>q(U)$. This contradicts the minimality of $U^{*}$.

Next we focus on the case of $1 \leq k \leq n-4$.

Let $\mathcal{U}_{1}(n, k)$ be the subset of $\mathcal{U}(n, k)$ in which the unicyclic graphs are obtained from $C_{g}$ by attaching $k$ paths at vertex $v_{0}$. Let $\mathcal{U}_{2}(n, k)$ be the subset of $\mathcal{U}(n, k)$ in which the unicyclic graphs are obtained from $P_{l+1}: v_{0} u_{1} \cdots u_{l}(l \geq 1)$ by attaching $C_{g}$ to one end vertex $v_{0}$ and $k$ paths to the other end vertex $u_{l}$ (see Fig. 3.1).

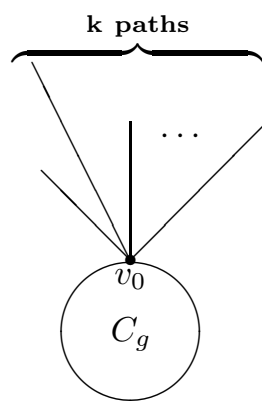

$\mathcal{U}_{1}(n, k)$

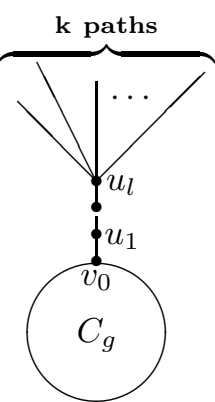

$\mathcal{U}_{2}(n, k)$

FIG. 3.1. Two classes of graphs in $\mathcal{U}(n, k)$ with girth $g$.

Let $\tilde{U}$ be the non-bipartite unicyclic graph obtained from $C_{g}$ by attaching $k$ pendant edges at vertex $v_{0}$. Clearly $\tilde{U} \in \mathcal{U}_{1}(n, k)$.

LEMma 3.2. For each non-bipartite unicyclic graph $U \in \mathcal{U}(n, k)$, either $q(U) \geq$ $q(\tilde{U})$ or there exists a graph $U_{1} \in \mathcal{U}_{2}(n, k)$ such that $q(U) \geq q\left(U_{1}\right)$.

Proof. Let $C_{g}$ be the unique odd cycle in $U$, where $V\left(C_{g}\right)=\left\{v_{0}, v_{1}, \ldots, v_{g-1}\right\}$. The unicyclic graph $U$ can be viewed as planting some tree $T_{i}$ at vertex $v_{i}$, where $0 \leq i \leq g-1$. Let $X$ be a unit $Q$-eigenvector of $U$ corresponding to $q(U)$. Without loss of generality, let $\left|x_{v_{0}}\right|=\max \left\{\left|x_{v_{i}}\right|: 0 \leq i \leq g-1\right\}$. Let $U_{0}$ be the graph obtained from $C_{g}$ by planting $T_{0}, T_{1}, \ldots, T_{g-1}$ (possibly trivial) at vertex $v_{0}$ to form a new big tree $T$ with root $v_{0}$, where $d\left(v_{0}\right) \geq 3$. From a repeated use of Lemma 2.2, we have $q(U) \geq q\left(U_{0}\right)$. Consider the graph $U_{0}$. Let $t$ be the cardinality of the vertices whose degrees are no less than 3 in $V(T) \backslash\left\{v_{0}\right\}$ and $X^{\prime}$ be a unit $Q$-eigenvector of $U_{0}$ 
corresponding to $q\left(U_{0}\right)$, and now we distinguish the following three cases:

Case 1. $t=0$. In this case, $U_{0} \in \mathcal{U}_{1}(n, k)$.

Case 1.1. $U_{0}$ is isomorphic to $\tilde{U}$, then $q\left(U_{0}\right)=q(\tilde{U})$.

Case 1.2. $U_{0}$ is not isomorphic to $\tilde{U}$, then there exists a vertex $v \in V(T) \backslash$ $\left\{v_{0}\right\}$ with degree 2 in $U_{0}$. By Lemmas 2.4 and 2.5. $\left|x_{v}^{\prime}\right| \geq\left|x_{v_{0}}^{\prime}\right|$. Denote $N\left(v_{0}\right)=$ $\left\{v_{1}, v_{g-1}, w_{1}, w_{2}, \ldots, w_{p}\right\}$. Assume that $w_{1}$ (possibly $v$ ) belongs to the unique path joining $v_{0}$ and $v$. Define $U_{1}=U_{0}-\left\{v_{0} w_{2}, v_{0} w_{3}, \ldots, v_{0} w_{p}\right\}+\left\{v w_{2}, v w_{3}, \ldots, v w_{p}\right\}$, clearly $U_{1} \in \mathcal{U}_{2}(n, k)$ and by Lemma 2.2, $q\left(U_{0}\right) \geq q\left(U_{1}\right)$.

Case 2. $t=1$. We can assume that there exists one vertex $v \in V(T) \backslash\left\{v_{0}\right\}$ with $d(v) \geq 3$ in $U_{0}$, then there is a unique path with length at least 1 joining $v_{0}$ and $v$. By Lemmas 2.4 and 2.5, $\left|x_{v}^{\prime}\right| \geq\left|x_{v_{0}}^{\prime}\right|$.

Case 2.1. $d\left(v_{0}\right) \geq 4$. Denote $N\left(v_{0}\right)=\left\{v_{1}, v_{g-1}, w_{1}, w_{2}, \ldots, w_{p}\right\}$. Assume that vertex $w_{1}$ belongs to the unique path joining $v_{0}$ and $v$. Define $U_{1}=U_{0}-$ $\left\{v_{0} w_{2}, v_{0} w_{3}, \ldots, v_{0} w_{p}\right\}+\left\{v w_{2}, v w_{3}, \ldots, v w_{p}\right\}$, clearly $U_{1} \in \mathcal{U}_{2}(n, k)$ and by Lemma 2.2, $q\left(U_{0}\right) \geq q\left(U_{1}\right)$.

Case 2.2. $d\left(v_{0}\right)=3$. In this case, $U_{1}=U_{0} \in \mathcal{U}_{2}(n, k)$, and $q\left(U_{0}\right)=q\left(U_{1}\right)$.

Case 3. $t>1$. Suppose that $u, v \in V(T) \backslash\left\{v_{0}\right\}$ are two vertices of $U_{0}$ whose degrees are 3 or greater, and $\left|x_{u}^{\prime}\right| \geq\left|x_{v}^{\prime}\right|$. Since $T$ is a tree, there is a path between $u$ and $v$ and only one neighbor of $v$, say $w$, is on the path. Assume that $\left\{v_{1}, v_{2}, \ldots, v_{d_{v}-2}\right\} \subset N(v) \backslash\{w\}$. Delete the edges $\left(v, v_{i}\right)$ and insert the edges $\left(u, v_{i}\right)\left(i=1,2, \ldots, d_{v}-2\right)$, then we get a new unicycle graph $U_{1}^{\prime}$. Obviously $U_{1}^{\prime}$ still has $k$ pendant vertices. By Lemma 2.2, we have $q\left(U_{0}\right) \geq q\left(U_{1}^{\prime}\right)$ and the cardinality of the vertices of degree 3 or greater decreases to $t-1$.

If $t-1>1$, to $U_{1}^{\prime}$ repeat the above step until the cardinality is reduced to one. So we get non-bipartite unicyclic graphs

$$
U_{2}^{\prime}, U_{3}^{\prime}, \ldots, U_{t-1}^{\prime}
$$

and

$$
q\left(U_{1}^{\prime}\right) \geq q\left(U_{2}^{\prime}\right) \geq \cdots \geq q\left(U_{t-1}^{\prime}\right)
$$

Moreover, each $U_{i}^{\prime}$ has $k$ pendant vertices. Refering to case 2, we always have $U_{1} \in \mathcal{U}_{2}(n, k)$ and $q\left(U_{t-1}^{\prime}\right) \geq q\left(U_{1}\right)$.

LEMma 3.3. Let $U^{*}$ have the minimal least $Q$-eigenvalue in $\mathcal{U}(n, k)$, where $1 \leq k \leq n-4$. Then $g\left(U^{*}\right)=3$. 
Proof. According to Lemma 3.2 we may assume that $U^{*} \in \mathcal{U}_{2}(n, k)$ or $U^{*}$ is isomorphic to $\tilde{U}$.

First consider the case of $U^{*} \in \mathcal{U}_{2}(n, k)$. Let $C_{g}$ be the unique odd cycle in $U^{*}$. Suppose that $g\left(U^{*}\right) \geq 5$, and there must exist at least four vertices $v_{i-1}, v_{i}, v_{i+1}, v_{i+2}$ of $C_{g}$ in $U^{*}$.

Let $X$ be a unit $Q$-eigenvector of $U^{*}$ corresponding to $q\left(U^{*}\right)$. Since $\left|x_{v_{0}}\right|=$ $\max \left\{\left|x_{v_{i}}\right|: 0 \leq i \leq g-1\right\}$, Lemma 2.4 implies $x_{v_{0}} \neq 0$. According to Lemma 2.6 (i), there must exist an edge $v_{i} v_{i+1} \in C_{g}$ such that $x_{v_{i}} x_{v_{i+1}} \geq 0$. Since $\left|x_{v_{0}}\right|=$ $\max \left\{\left|x_{v_{i}}\right|: 0 \leq i \leq g-1\right\}$, by Lemma 2.6 (iv), $v_{i} v_{i+1} \neq v_{0} v_{1}$ and $v_{i} v_{i+1} \neq v_{g-1} v_{0}$, i.e., $1 \leq i \leq g-2$. Without loss of generality, we can assume that $\left|x_{v_{i}}\right| \leq\left|x_{v_{i+1}}\right|$, by Lemma 2.6 $(i i i)$, then $x_{v_{i+1}} \neq 0$. Now we distinguish the following three cases:

Case 1. $\left(x_{v_{i-1}}-x_{v_{i+2}}\right)\left(2 x_{v_{i}}+x_{v_{i-1}}+x_{v_{i+2}}\right) \geq 0$.

Case 1.1. $i=1$. That is to say, $\left(x_{v_{0}}-x_{v_{3}}\right)\left(2 x_{v_{1}}+x_{v_{0}}+x_{v_{3}}\right) \geq 0$. Deleting the edge $v_{1} v_{0}$ and inserting the edge $v_{1} v_{3}$, we can get a new graph $U \in \mathcal{U}_{2}(n, k)$, and

$$
\begin{aligned}
q\left(U^{*}\right)-q(U) & \geq X^{T} Q\left(U^{*}\right) X-X^{T} Q(U) X \\
& =\left(x_{v_{0}}+x_{v_{1}}\right)^{2}-\left(x_{v_{1}}+x_{v_{3}}\right)^{2} \\
& =\left(x_{v_{0}}-x_{v_{3}}\right)\left(2 x_{v_{1}}+x_{v_{0}}+x_{v_{3}}\right) \\
& \geq 0 .
\end{aligned}
$$

The equality $q\left(U^{*}\right)=q(U)=q$ holds only if $X$ is also a unit least $Q$-eigenvector of $U$ and $\left(x_{v_{0}}-x_{v_{3}}\right)\left(2 x_{v_{1}}+x_{v_{0}}+x_{v_{3}}\right)=0$. By the eigenvalue equations (2.1) of vertices $v_{0}$ and $v_{1}$ in both $U^{*}$ and $U$, we have

$$
\begin{array}{ll}
(q-3) x_{v_{0}}=x_{u_{1}}+x_{v_{1}}+x_{v_{g-1}}, & (q-2) x_{v_{1}}=x_{v_{0}}+x_{v_{2}}, \\
(q-2) x_{v_{0}}=x_{u_{1}}+x_{v_{g-1}}, & (q-2) x_{v_{1}}=x_{v_{2}}+x_{v_{3}} .
\end{array}
$$

Hence, $x_{v_{1}}=-x_{v_{0}}=-x_{v_{3}}$. By Lemma 2.6 (iv), $\left|x_{v_{3}}\right|>\left|x_{v_{1}}\right|$, a contradiction. Hence, we find a graph $U \in \mathcal{U}_{2}(n, k)$ such that $q\left(U^{*}\right)>q(U)$. This contradicts the minimality of $U^{*}$.

Case 1.2. $2 \leq i \leq g-2$. Define $\hat{U}=U^{*}-v_{i} v_{i-1}+v_{i} v_{i+2}$, and

$$
\begin{aligned}
q\left(U^{*}\right)-q(\hat{U}) & \geq X^{T} Q\left(U^{*}\right) X-X^{T} Q(\hat{U}) X \\
& =\left(x_{v_{i}}+x_{v_{i-1}}\right)^{2}-\left(x_{v_{i}}+x_{v_{i+2}}\right)^{2} \\
& =\left(x_{v_{i-1}}-x_{v_{i+2}}\right)\left(2 x_{v_{i}}+x_{v_{i-1}}+x_{v_{i+2}}\right) \\
& \geq 0 .
\end{aligned}
$$


At this time, vertex $v_{i-1}$ is a new pendant vertex in $\hat{U}$. Note that $k \geq 1$, there exists an old pendant vertex $v$ in $\hat{U}$. Let $U=\hat{U}-v_{1} v_{0}+v_{1} v$, clearly $U \in \mathcal{U}_{2}(n, k)$. Since $\left|x_{v_{0}}\right|<\left|x_{v}\right|$, by Lemma 2.2, $q(\hat{U})>q(U)$. This contradicts the minimality of $U^{*}$.

Case 2. $\left(x_{v_{i+2}}-x_{v_{i-1}}\right)\left(2 x_{v_{i+1}}+x_{v_{i-1}}+x_{v_{i+2}}\right) \geq 0$.

Case 2.1. $i=g-2$. That is, $\left(x_{v_{0}}-x_{v_{g-3}}\right)\left(2 x_{v_{g-1}}+x_{v_{0}}+x_{v_{g-3}}\right) \geq 0$. Deleting the edge $v_{g-1} v_{0}$ and inserting the edge $v_{g-1} v_{g-3}$, we get a new graph $U \in \mathcal{U}_{2}(n, k)$, and

$$
\begin{aligned}
q\left(U^{*}\right)-q(U) & \geq X^{T} Q\left(U^{*}\right) X-X^{T} Q(U) X \\
& =\left(x_{v_{0}}+x_{v_{g-1}}\right)^{2}-\left(x_{v_{g-1}}+x_{v_{g-3}}\right)^{2} \\
& =\left(x_{v_{0}}-x_{v_{g-3}}\right)\left(2 x_{v_{g-1}}+x_{v_{0}}+x_{v_{g-3}}\right) \\
& \geq 0
\end{aligned}
$$

The equality $q\left(U^{*}\right)=q(U)=q$ holds only if $X$ is also a unit least $Q$-eigenvector of $U$ and $\left(x_{v_{0}}-x_{v_{g-3}}\right)\left(2 x_{v_{g-1}}+x_{v_{0}}+x_{v_{g-3}}\right)=0$. By the eigenvalue equations (2.1) of vertices $v_{0}$ and $v_{g-1}$ in both $U^{*}$ and $U$, we have

$$
\begin{array}{ll}
(q-3) x_{v_{0}}=x_{u_{1}}+x_{v_{1}}+x_{g-1}, & (q-2) x_{v_{g-1}}=x_{v_{0}}+x_{v_{g-2}}, \\
(q-2) x_{v_{0}}=x_{u_{1}}+x_{v_{1}}, & (q-2) x_{v_{g-1}}=x_{v_{g-3}}+x_{v_{g-2}} .
\end{array}
$$

Hence, $x_{v_{g-1}}=-x_{v_{0}}=-x_{v_{g-3}}$. By Lemma 2.6 (iv), $\left|x_{v_{g-3}}\right|>\left|x_{v_{g-1}}\right|$, a contradiction. Hence, we find a graph $U \in \mathcal{U}_{2}(n, k)$ such that $q\left(U^{*}\right)>q(U)$. This contradicts the minimality of $U^{*}$.

Case 2.2. $1 \leq i \leq g-3$. Define $\hat{U}=U^{*}-v_{i+1} v_{i+2}+v_{i+1} v_{i-1}$, and

$$
\begin{aligned}
q\left(U^{*}\right)-q(\hat{U}) & \geq X^{T} Q\left(U^{*}\right) X-X^{T} Q(\hat{U}) X \\
& =\left(x_{v_{i+1}}+x_{v_{i+2}}\right)^{2}-\left(x_{v_{i+1}}+x_{v_{i-1}}\right)^{2} \\
& =\left(x_{v_{i+2}}-x_{v_{i-1}}\right)\left(2 x_{v_{i+1}}+x_{v_{i-1}}+x_{v_{i+2}}\right) \\
& \geq 0
\end{aligned}
$$

At this time, vertex $v_{i+2}$ is a new pendant vertex in $\hat{U}$. Note that $k \geq 1$, there exists an old pendant vertex $v$ in $\hat{U}$. Let $U=\hat{U}-v_{g-1} v_{0}+v_{g-1} v$, clearly $U \in \mathcal{U}_{2}(n, k)$. Since $\left|x_{v_{0}}\right|<\left|x_{v}\right|$, by Lemma 2.2. $q(\hat{U})>q(U)$. Hence, we find a graph $U \in \mathcal{U}_{2}(n, k)$ such that $q\left(U^{*}\right)>q(U)$. This contradicts the minimality of $U^{*}$.

Case 3. $\left(x_{v_{i-1}}-x_{v_{i+2}}\right)\left(2 x_{v_{i}}+x_{v_{i-1}}+x_{v_{i+2}}\right)<0, \quad\left(x_{v_{i+2}}-x_{v_{i-1}}\right)\left(2 x_{v_{i+1}}+x_{v_{i-1}}+\right.$ $\left.x_{v_{i+2}}\right)<0$. Hence, $2 x_{v_{i}}+x_{v_{i-1}}+x_{v_{i+2}}$ and $2 x_{v_{i+1}}+x_{v_{i-1}}+x_{v_{i+2}}$ are of opposite sign. Since we assume that $\left|x_{v_{i}}\right| \leq\left|x_{v_{i+1}}\right|$, we have the following two cases: 
Case 3.1. $x_{v_{i+1}} \geq x_{v_{i}} \geq 0$. Hence, $2 x_{v_{i+1}}+x_{v_{i-1}}+x_{v_{i+2}}>0$, by Lemma 2.6 (ii), $x_{v_{i-1}}, x_{v_{i+2}} \leq 0$, and $x_{v_{i+1}}>\frac{1}{2}\left(\left|x_{v_{i-1}}\right|+\left|x_{v_{i+2}}\right|\right) \geq \min \left\{\left|x_{v_{i-1}}\right|,\left|x_{v_{i+2}}\right|\right\}$, which is a contradiction to Lemma 2.6 (iv).

Case 3.2. $x_{v_{i+1}} \leq x_{v_{i}} \leq 0$. It follows that $2 x_{v_{i+1}}+x_{v_{i-1}}+x_{v_{i+2}}<0$, by Lemma $2.6(i i), x_{v_{i-1}}, x_{v_{i+2}} \geq 0$, and $\left|x_{v_{i+1}}\right|>\frac{1}{2}\left(x_{v_{i-1}}+x_{v_{i+2}}\right) \geq \min \left\{x_{v_{i-1}}, x_{v_{i+2}}\right\}$, which is a contradiction to Lemma 2.6 (iv).

At last, we consider the case of $U^{*}$ is isomorphic to $\tilde{U}$.

If $U^{*}$ is isomorphic to $\tilde{U}$, we have $g\left(U^{*}\right) \geq 5$, since $k+g\left(U^{*}\right)=n \geq k+4$. Similar to the above proof with $U^{*} \in \mathcal{U}_{2}(n, k)$, we can always find at least four vertices $v_{i-1}, v_{i}, v_{i+1}, v_{i+2}$ of $C_{g}$ in $U^{*}$ and obtain a contradiction in each case. Hence, it is impossible that $U^{*}$ is isomorphic to $\tilde{U}$.

Let $\mathcal{U}^{*}(n, k)$ be the set of non-bipartite unicyclic graphs in $\mathcal{U}_{2}(n, k)$ which have girth 3 (see Fig. 3.2).

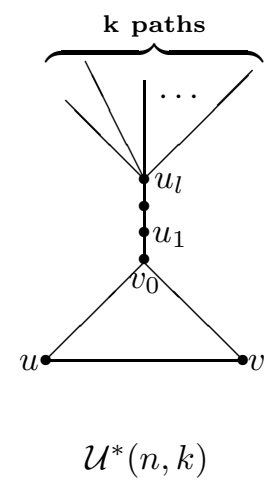

FIG. 3.2. The class of graphs in $\mathcal{U}_{2}(n, k)$ with girth 3 .

TheOREM 3.4. Let $U^{*}$ have the minimal least $Q$-eigenvalue in $\mathcal{U}(n, k)$, where $1 \leq k \leq n-4$. Then $U^{*}$ is isomorphic to $\triangle_{n}^{k}$.

Proof. According to Lemma 3.3. we can assume without loss of generality that $U^{*} \in \mathcal{U}^{*}(n, k)$. If $U^{*}$ is not isomorphic to $\triangle_{n}^{k}$, then $k \geq 2$ and there exists at least a pendant path with length 2 or greater at vertex $u_{l}$ in $U^{*}$. Let $X$ be a unit $Q$-eigenvector of $U^{*}$ corresponding to $q\left(U^{*}\right)$. From a repeated use of Lemma 2.3. we obtain a new graph $U^{\prime} \in \mathcal{U}^{*}(n, k)$ which has one pendant path: $u_{l} z_{1} z_{2} \cdots z_{p-1} z_{p}$ with length $p=n-k-l-2$ and $k-1$ pendant paths with length 1 at vertex $u_{l}$ in $U^{\prime}$. Note that $x_{u_{l}} \neq 0$, by Lemma 2.3. $q\left(U^{*}\right)>q\left(U^{\prime}\right)$.

Consider unicyclic graph $U^{\prime}$, let $X^{\prime}$ be a unit $Q$-eigenvector of $U^{\prime}$ corresponding to $q\left(U^{\prime}\right)$. Denote $N\left(u_{l}\right) \backslash\left\{u_{l-1}, z_{1}\right\}=\left\{w_{1}, w_{2}, \ldots, w_{k-1}\right\}$. Then $\triangle_{n}^{k}=U^{\prime}-w_{i} u_{l}+$ 
$w_{i} z_{p-1}$, where $1 \leq i \leq k-1$. Note that $\left|x_{u_{l}}^{\prime}\right|<\left|x_{z_{p-1}}^{\prime}\right|$, by Lemma 2.2 then $q\left(U^{\prime}\right)>$ $q\left(\triangle_{n}^{k}\right)$. Hence, we get $q\left(U^{*}\right)>q\left(\triangle_{n}^{k}\right)$. By the definition of $U^{*}$, then $U^{*}$ is isomorphic to $\triangle_{n}^{k}$.

TheOREM 3.5. Let $1 \leq k \leq n-4$. Then $q\left(\triangle_{n}^{k}\right)<q\left(\triangle_{n}^{k+1}\right)$.

Proof. Consider the unicyclic graph $\triangle_{n}^{k+1}$. Let $X$ be a unit $Q$-eigenvector of $\triangle_{n}^{k+1}$ corresponding to $q\left(\triangle_{n}^{k+1}\right)$. Denote $k+1$ pendant vertices by $v_{1}, v_{2}, \ldots, v_{k}, v_{k+1}$ and their common neighbor $u_{l}$. Then $\triangle_{n}^{k}=\triangle_{n}^{k+1}-v_{i} u_{l}+v_{i} v_{k+1}$, where $1 \leq i \leq k$. Note that $\left|x_{u_{l}}\right|<\left|x_{v_{k+1}}\right|$, by Lemma 2.2. we have $q\left(\triangle_{n}^{k+1}\right)>q\left(\triangle_{n}^{k}\right)$.

Theorem 1.1 follows naturally from Theorems 3.1, 3.4 and 3.5.

Let $\mathcal{U}_{n}$ be the set of non-bipartite unicyclic graphs on $n$ vertices, where $n \geq 4$. Then $\mathcal{U}_{n}=\left\{C_{n}\right\} \cup \mathcal{U}(n, 1) \cup \mathcal{U}(n, 2) \cup \cdots \cup \mathcal{U}(n, n-3)$.

Lemma 3.6. Let $U^{* *}$ have the minimal least $Q$-eigenvalue in $\mathcal{U}_{n}$, where $n \geq 4$. Then $U^{* *}$ is not isomorphic to $C_{n}$.

Proof. By way of contradiction, suppose that $U^{* *}$ is isomorphic to $C_{n}$, then we have $n \geq 5$ is odd. Let $X$ be a unit $Q$-eigenvector of $U^{* *}$ corresponding to $q\left(U^{* *}\right)$. Using the same techniques as the proof of Lemma 3.3, we can always find at least four vertices $v_{i-1}, v_{i}, v_{i+1}, v_{i+2}$ of $C_{n}$ and obtain a contradiction in each case, since $\left|x_{v_{i}}\right| \neq\left|x_{v_{i-1}}\right|$ and $\left|x_{v_{i+1}}\right| \neq\left|x_{v_{i+2}}\right|$. Hence, $U^{* *}$ is not isomorphic to $C_{n}$. $\mathrm{Q}$

According to Lemma 3.6, then $U^{* *} \in \mathcal{U}(n, 1) \cup \mathcal{U}(n, 2) \cup \cdots \cup \mathcal{U}(n, n-3)$. As an immediate consequence of Theorems 1.1, we have the following Corollary 3.7, which is one of the main results in [2].

Corollary 3.7. ([2]) Let $\mathcal{U}_{n}$ be the set of non-bipartite unicyclic graphs on $n$ vertices, where $n \geq 4$. For any $U \in \mathcal{U}_{n}$, we have

$$
q(U) \geq q\left(\triangle_{n}^{1}\right)
$$

with equality if and only if $U$ is isomorphic to $\triangle_{n}^{1}$.

Acknowledgment. The authors would like to thank the anonymous referees very much for valuable suggestions and corrections which improved the paper.

\section{REFERENCES}

[1] R.A. Brualdi and E.S. Solheid. On the spectral radius of complementary acyclic matrices of zeros and ones. SIAM J. Algebraic Discrete Methods, 7:265-272, 1986.

[2] D.M. Cardoso, D. Cvetković, P. Rowlinson, and S. Simić. A sharp lower bound for the least eigenvalue of the signless Laplacian of a non-bipartite graph. Linear Algebra Appl., 429:27702780,2008 . 
[3] D. Cvetković, P. Rowlinson, and S. Simić. Signless Laplacian of finite graphs. Linear Algebra Appl., 423:155-171, 2007.

[4] E.R. van Dam and W.H. Haemers. Which graphs are determined by their spectrum? Linear Algebra Appl., 373:241-272, 2003.

[5] K.Ch. Das. On conjectures involving second largest signless Laplacian eigenvalue of graphs. Linear Algebra Appl., 432:3018-3029, 2010.

[6] Y.Z. Fan, S.C. Gong, Y. Wang, and Y.B. Gao. First eigenvalue and first eigenvectors of a nonsingular unicyclic mixed graph. Discrete Math., 309:2479-2487, 2009.

[7] W. Haemers and E. Spence. Enumeration of cospectral graphs. European J. Combin., 25:199$211,2004$.

[8] S.C. Li and S.J. Wang. The least eigenvalue of the signless Laplacian of the completments of trees. Linear Algebra Appl., 436:2398-2405, 2012.

[9] Y. Wang and Y.Z. Fan. The least eigenvalue of signless Laplacian of graphs under perturbation. Linear Algebra Appl., 436:2084-2092, 2012. 